\title{
A CONDUTA GERENCIAL DA ENFERMEIRA: UM ESTUDO FUNDAMENTADO NAS TEORIAS GERAIS DA ADMINISTRAÇÃO
}

\author{
Marcia Simoni Fernandes ${ }^{1}$ \\ Carla Aparecida Spagnol ${ }^{2}$ \\ Maria Auxiliadora Trevizan ${ }^{3}$ \\ Miyeko Hayashida ${ }^{4}$
}

Fernandes MS, Spagnol CA, Trevizan MA, Hayashida M. A conduta gerencial da enfermeira: um estudo fundamentado nas teorias gerais da administração. Rev Latino-am Enfermagem 2003 março-abril; 11(2):161-7.

O objetivo desta pesquisa foi identificar a conduta gerencial da enfermeira em uma maternidade privada do interior paulista. Para a coleta de dados utilizamos questionário com 20 proposições relativas ao trabalho das enfermeiras que atuam nos diversos setores do hospital. Realizamos análise estatística descritiva dos dados obtidos. Os resultados mostraram tendência à democratização na conduta das enfermeiras investigadas, pois a maioria das respostas privilegiou questões acerca do trabalho em equipe, participação dos trabalhadores e desenvolvimento grupal. Evidenciamos, ainda, que grande parte das respondentes discordou das proposições que abordavam os princípios norteadores da Teoria Clássica da Administração.

DESCRITORES: enfermagem, gerência, hospitais

\section{NURSES MANAGERIAL CONDUCT: A STUDY BASED ON ADMINISTRATION GENERAL THEORIES}

The purpose of this study was to identify nurses managerial conduct in a private maternity hospital located in the interior of the São Paulo state, Brazil. In order to collect data, authors used a questionnaire with 20 propositions related to the work of nurses in the different units of the hospital. Following, authors performed a descriptive statistical analysis of the data. Results showed a tendency toward democratization in the conduct of the nurses investigated as the majority of the responses privileged questions on team work, workers' participation and group development. Also, authors evidenced that great part of the responses did not agree with the propositions about the principles of the Classical Administration Theory.

DESCRIPTORS: nursing, management, hospital

\section{LA CONDUCTA GERENGIAL DE LA ENFERMERA: UN ESTUDIO BASADO EN LAS TEORÍAS GENERALES DE LA ADMINISTRACIÓN}

El objetivo de ésta investigación fue identificar la conducta gerencial de la enfermera en una unidad obstétrica del interior paulista. Para la recolección de datos utilizamos un cuestionario con 20 enunciados relacionados con el trabajo de las enfermeras que actúan en los diversos sectores del hospital. Realizamos un análisis descriptivo de los datos obtenidos. Los resultados mostraron una tendencia a la democratización en la conducta de las enfermeras investigadas, pues la mayoría de las respuestas privilegiaron aspectos relacionados con el trabajo en equipo, participación de los trabajadores y desarrollo grupal. Es evidente aún, que gran parte de las que respondieron estuvo en desacuerdo con los enunciados que abordaban los principios orientadores de la Teoría Clásica de la Administración.

DESCRIPTORES: enfermería, gerencia, hospitales

\footnotetext{
${ }^{1}$ Enfermeira, Professor do Departamento de Enfermagem da Universidade de Caxias do Sul, Mestre em Enfermagem; ${ }^{2}$ Enfermeira, Professor Assistente da Escola de Enfermagem da Universidade Federal de Minas Gerais, Mestre em Enfermagem, e-mail: spagnol@enf.ufmg.br; ${ }^{3}$ Enfermeira, Professor Titular; ${ }^{4}$ Enfermeira, Doutor em Enfermagem, Responsável pela Seção de Apoio Laboratorial. Escola de Enfermagem de Ribeirão Preto da Universidade de São Paulo, Centro Colaborador da OMS para o desenvolvimento da pesquisa em enfermagem
} 
INTRODUÇÃO

$H$ istoricamente as enfermeiras têm adotado princípios da Escola Científica e Clássica da Administração para gerenciarem o seu trabalho, tendo em vista a estruturação e organização do Serviço de Enfermagem nas instituições de saúde. Notamos que, atualmente, algumas características desse estilo de gerência como: a fragmentação das atividades, a impessoalidade nas relações, a centralização do poder e a rígida hierarquia ainda são marcantes no cotidiano do trabalho da enfermagem, demonstrando nitidamente a conduta gerencial autoritária que as enfermeiras têm adotado.

Ao longo da nossa trajetória profissional, observamos que a enfermeira tem desempenhado nítido papel de controladora do trabalho dos demais elementos da equipe de enfermagem, sendo vista apenas como uma profissional que determina e checa as atividades a serem executadas. Ela tem centrado o seu trabalho predominantemente em atividades administrativas burocráticas, distanciando-se, dessa forma, de sua função precípua que é o gerenciamento do cuidado a ser prestado ao paciente ${ }^{(1)}$.

A busca do cliente por produtos e serviços de qualidade, o crescente avanço tecnológico em diversas áreas, mudanças nos processos de trabalho, problemas financeiros, entre outros, são fatores que estão exigindo das empresas públicas e privadas adaptação rápida e constante às mudanças e instabilidades dos tempos atuais. Nesse contexto, observamos que a conduta do gerente deve ser modificada perante as demandas contemporâneas.

Frente a essas mudanças, alguns hospitais estão buscando novos modelos assistenciais e novas formas de gestão, a fim de alcançarem resultados capazes de melhorar o bem-estar dos indivíduos e da comunidade, humanizar a assistência, otimizar recursos e garantir a qualidade dos serviços prestados.

Dessa forma, acreditamos que a enfermagem deverá também acompanhar as transformações da sociedade contemporânea. Portanto, cabe às enfermeiras, que são responsáveis pela gerência do cuidado, nessas instituições que estão acompanhando as mudanças, buscarem cada vez mais inovações na esfera gerencial que permitam amenizar as conseqüências do modelo de gerência clássica, adotado até hoje na maioria das instituições de saúde.
Para esse remodelamento da profissão, torna-se necessária a integração de novos conhecimentos e habilidades, sintonizados a uma prática administrativa mais aberta, mais flexível e participativa, fundamentada não só na razão, mas também na sensibilidade e na intuição ${ }^{(2)}$.

Diante dessas considerações, elaboramos a seguinte questão como norteadora do nosso estudo: que conduta gerencial a enfermeira vem assumindo frente às mudanças que estão ocorrendo nos dias atuais?

O objetivo desta pesquisa é identificar a conduta gerencial da enfermeira em uma maternidade privada localizada no interior do Estado de São Paulo.

Este estudo se justifica através da seguinte premissa: conhecendo a conduta gerencial da enfermeira nessa instituição, poderemos estar contribuindo para a reflexão acerca da gerência em enfermagem na perspectiva de encontrarmos aspectos relevantes para a construção de nova prática profissional.

\section{METODOLOGIA}

O presente estudo foi desenvolvido em uma maternidade privada, localizada no interior de São Paulo. Trata-se de instituição com 86 leitos, distribuídos em duas unidades de internação obstétrica e ginecológica, uma unidade pediátrica, berçário e unidade de terapia intensiva neonatal. Além disso, possui centro cirúrgico e obstétrico, central de material, e serviços auxiliares de diagnóstico e tratamento. Presta assistência a pacientes particulares e conveniados com vários planos de saúde.

Participaram do estudo 12 enfermeiras, das 14 que compõem o quadro de pessoal de enfermagem do hospital em estudo. As enfermeiras investigadas atuam nos três turnos de trabalho (manhã, tarde e noite) em diversos setores.

Para a coleta de dados utilizamos um instrumento adaptado $^{(3)}$, contendo questões referentes aos dados de identificação das enfermeiras, bem como proposições que dizem respeito ao trabalho gerencial que desenvolvem, sendo subsidiadas pelas Teorias Administrativas, desde as mais tradicionais até as mais contemporâneas. Cada proposição possui quatro alternativas de respostas, conforme descritas abaixo.

\begin{tabular}{|c|c|c|c|}
\hline $\begin{array}{c}\text { Concordo } \\
\text { plenamente }\end{array}$ & $\begin{array}{c}\text { Mais concordo } \\
\text { que discordo }\end{array}$ & $\begin{array}{c}\text { Mais discordo } \\
\text { que concordo }\end{array}$ & $\begin{array}{c}\text { Discordo } \\
\text { plenamente }\end{array}$ \\
\hline
\end{tabular}


Para a validação do instrumento de coleta de dados selecionamos três juízes, que atuam na docência na área de Administração em Enfermagem, analisaram o conteúdo, a clareza dos itens, a compreensão e a forma de apresentação do mesmo. Todas as sugestões foram acatadas por terem sido bastante pertinentes, garantindo melhor entendimento acerca das questões.

Entregamos às enfermeiras um questionário, ressaltando que respondessem apenas uma das alternativas, não deixando respostas em branco, e que, em caso de dúvidas, consultassem as autoras. Dos 14 questionários distribuídos, 12 foram respondidos e 2 devolvidos em branco. Os questionários foram aplicados mediante aprovação do hospital e o consentimento das enfermeiras, através do seu aceite no Termo de Consentimento Pós-Informação, garantindo às informantes o sigilo e o anonimato das respostas.

Para analisar os dados, atribuímos um valor numérico (escore) a cada possibilidade de resposta, sendo que quanto maior o escore mais as enfermeiras estariam discordando da proposição elaborada e quanto menor fosse esse valor atribuído mais estariam concordando com a afirmativa. Os valores atribuídos variaram de 1 a 4 , representados na figura abaixo.

\begin{tabular}{|c|c|c|c|}
\hline 1 & 2 & 3 & 4 \\
\hline $\begin{array}{c}\text { Concordo } \\
\text { plenamente }\end{array}$ & $\begin{array}{c}\text { Mais concordo } \\
\text { que discordo }\end{array}$ & $\begin{array}{c}\text { Mais discordo } \\
\text { que concordo }\end{array}$ & $\begin{array}{c}\text { Discordo } \\
\text { plenamente }\end{array}$ \\
\hline
\end{tabular}

Ressaltamos que foi realizada análise estatística descritiva dos dados obtidos, utilizando como subsídio o referencial da Teoria Geral da Administração.

\section{APRESENTAÇÃO DOS DADOS}

Caracterização da população

A primeira parte do instrumento de coleta de dados permitiu-nos caracterizar as participantes da pesquisa, em relação a: idade, tempo de término de curso, cursos realizados de pós-graduação e tempo de trabalho na instituição em estudo.

A média de idade das participantes foi de 33,3 anos. Os dados mostraram ainda que duas enfermeiras possuem tempo inferior a 3 anos de formadas, quatro possuem de 3 a 8 anos, duas de 9 a 14 anos, enquanto que outras quatro de 15 a 20 anos. Observamos que das
12 enfermeiras somente 3 realizaram cursos de pósgraduação, sendo que, dessas, duas especializaram-se em enfermagem do trabalho e uma obteve o título de especialista em enfermagem obstétrica. Cumpre salientar que uma enfermeira possui formação em licenciatura em enfermagem.

Os dados evidenciaram também que as enfermeiras possuem tempo de serviço na instituição variando de 1 a 15 anos.

Análise dos dados

A Tabela 1 mostra a somatória, a média e o desvio padrão dos escores atribuídos pelas enfermeiras relativos às proposições elaboradas acerca da conduta gerencial das mesmas. Analisaremos as proposições que obtiveram a maior e a menor somatória dos escores.

Tabela 1 - Somatória, média e desvio padrão dos escores atribuídos pelas enfermeiras de uma maternidade privada, para cada uma das proposições elaboradas relacionadas à conduta gerencial das mesmas. Ribeirão Preto-SP, 1998

\begin{tabular}{cccc}
\hline Proposições & Somatória & Média & Desvio Padrão \\
\hline 01 & 21 & 1,750 & 0,754 \\
02 & 15 & 1,250 & 0,622 \\
03 & 23 & 1,917 & 0,515 \\
04 & 20 & 1,667 & 0,651 \\
$\mathbf{0 5}$ & $\mathbf{1 4}$ & $\mathbf{1 , 1 6 7}$ & $\mathbf{0 , 3 8 9}$ \\
$\mathbf{0 6}$ & $\mathbf{1 4}$ & $\mathbf{1 , 1 6 7}$ & $\mathbf{0 , 3 8 9}$ \\
$\mathbf{0 7}$ & $\mathbf{1 4}$ & $\mathbf{1 , 1 6 7}$ & $\mathbf{0 , 3 8 9}$ \\
08 & 32 & 2,667 & 0,651 \\
09 & 17 & 1,417 & 0,669 \\
10 & 29 & 2,417 & 1,084 \\
$\mathbf{1 1}$ & $\mathbf{4 0}$ & $\mathbf{3 , 3 3 3}$ & $\mathbf{0 , 7 7 8}$ \\
$\mathbf{1 2}$ & $\mathbf{1 4}$ & $\mathbf{1 , 1 6 7}$ & $\mathbf{0 , 3 8 9}$ \\
13 & 27 & 2,250 & 0,866 \\
14 & 22 & 1,833 & 0,937 \\
15 & 17 & 1,417 & 0,515 \\
16 & 34 & 2,833 & 1,030 \\
$\mathbf{1 7}$ & 14 & 1,167 & 0,389 \\
$\mathbf{1 8}$ & $\mathbf{3 8}$ & $\mathbf{3 , 1 6 7}$ & $\mathbf{0 , 9 3 7}$ \\
19 & 15 & 1,250 & 0,452 \\
$\mathbf{2 0}$ & $\mathbf{3 5}$ & $\mathbf{2 , 9 1 7}$ & $\mathbf{0 , 9 0 0}$ \\
\hline
\end{tabular}

A proposição 5 que versa sobre a presença de conflitos nas relações de trabalho, onde a enfermeira procura conhecer antecipadamente as causas e motivos dos problemas, visando solucioná-los juntamente com sua equipe, obteve média de 1,167 com desvio padrão de 0,389 dos escores atribuídos pelas respondentes, que concordaram com essa afirmativa. Esse resultado mostra atitude mais democrática da enfermeira com a sua equipe de trabalho, incentivando a participação de todos os 
elementos do grupo na resolução dos problemas encontrados no cotidiano. Acreditamos que tal atitude faça com que seus colaboradores sejam co-responsáveis pelo trabalho desenvolvido e não meros executores de tarefas determinadas.

Constatamos por meio da literatura que conceitos como motivação, dinâmica de grupo, comunicação, liderança, entre outros, foram desenvolvidos a partir da Escola das Relações Humanas, preconizada por Elton Mayo. Essa abordagem humanística iniciou uma fase das Teorias Administrativas que enfatizou as "pessoas" inseridas nas organizações, ao invés de focalizar "tarefas e estruturas"(4).

Ao longo do tempo, outras abordagens gerenciais foram surgindo como o gerenciamento da qualidade associado aos princípios da gerência participativa, introduzindo a idéia dos círculos de controle de qualidade (CCQ) como locais apropriados para a discussão dos problemas existentes, tendo como objetivo eliminar os conflitos e criar consensos acerca das formas de organização do trabalho ${ }^{(5)}$.

A gerência participativa e os programas de qualidade são abordagens gerenciais que preconizam, dentre outras, a descentralização das decisões e aproximação de todos os elementos da equipe de trabalho, oferecendo aos mesmos oportunidade de participarem efetivamente da discussão e aperfeiçoamento constantes do processo de trabalho. Portanto, a gerência participativa e o exercício da democracia são pontos fundamentais para o desenvolvimento do trabalho, pois acreditamos que, nessa forma de gerenciamento, os trabalhadores procuram melhorar a qualidade do produto final, bem como suas condições de trabalho.

Atualmente as empresas devem ser baseadas em equipes, diminuindo seus níveis hierárquicos, a fim de obterem responsabilidade compartilhada. Além disso, as equipes devem ter o máximo de autonomia para desenvolver novos projetos e métodos de trabalho, formular políticas de pessoal, bem como sugerir novas diretrizes para a organização ${ }^{(6)}$.

A organização do trabalho baseada na formação de equipes, "certamente tem sido a forma mais democrática, produtiva e humanizada de se efetuar o trabalho em saúde"(7). Pensando sob essa perspectiva, e preocupadas com o compromisso de todos os elementos da equipe com a assistência prestada aos clientes, acreditamos que cada vez mais as enfermeiras estarão adotando condutas norteadas pelo estilo de gerência participativa.

Entendemos que essas novas abordagens gerenciais podem estar influenciando a conduta das enfermeiras desse estudo, uma vez que a instituição, à qual estão inseridas, adotou os princípios e a filosofia do gerenciamento da qualidade.

Diante da afirmativa contida na proposição $6 \mathrm{em}$ que as enfermeiras devem sempre buscar atualização na sua área de conhecimento, bem como incentivar os seus colaboradores para adotarem esse tipo de ação, encontramos a média de 1,167 com desvio padrão de 0,389 .

Consideramos relevante a proposição acima, uma vez que a reciclagem dos profissionais é indispensável, diante das constantes mudanças organizacionais que atualmente vêm ocorrendo nas instituições de saúde. Assim, entendemos que a enfermeira necessita estar constantemente atualizada, influenciando seu grupo de maneira positiva, criando um ambiente que vise o crescimento individual, para que acompanhem a crescente incorporação de novas tecnologias, a mudança nos processos de trabalho e no perfil dos clientes, que estão ficando cada vez mais exigentes, buscando serviços de qualidade.

A proposição 7 aponta que as enfermeiras consideram importantes tanto os aspectos técnicos específicos do seu trabalho gerencial quanto aqueles relacionados ao desenvolvimento do grupo, obtendo média de 1,167 e desvio padrão de 0,389.

Nesse sentido, podemos inferir que as enfermeiras acreditam na importância do trabalho em equipe e na valorização dos colaboradores, entendendo-os como indivíduos competentes, criativos, e responsáveis pelo desenvolvimento do trabalho com qualidade.

O desenvolvimento dos trabalhadores é uma constante nas atuais abordagens gerenciais, as quais preconizam que a figura do gerente, enquanto líder da equipe, é essencial para impulsionar o desenvolvimento dos indivíduos, estimulando a criatividade e a satisfação no trabalho.

As enfermeiras responsáveis por liderarem a equipe de enfermagem necessitam desenvolver habilidades relativas à comunicação e ao relacionamento interpessoal, pois, para Senge, os líderes do futuro assumirão diferentes posturas no desenvolvimento de suas funções, destacando dentre outras o papel de professor e guia potencializador, 
auxiliando as pessoas a desenvolverem-se, propiciando o trabalho em grupo ${ }^{(8)}$.

Desenvolver um trabalho em grupo, portanto, que busca alcançar um objetivo comum - a assistência prestada ao paciente livre de riscos - é fundamental para que o enfermeiro possa exercer o seu papel de gerente perante a equipe de enfermagem ${ }^{(9)}$.

Os achados da proposição 12 revelaram média de 1,167 com desvio padrão de 0,389 , concordando que a enfermeira, ao planejar a assistência de enfermagem, considera importante ouvir o cliente e avaliar as suas necessidades, contando com a participação de todos da sua equipe. Esse resultado nos leva a pensar que as enfermeiras investigadas além de se preocuparem com o comprometimento da sua equipe nas atividades desenvolvidas, também estão buscando envolver os pacientes no seu processo terapêutico. A palavra participação é primordial para um novo estilo de gerência, mas não podemos esquecer que tal conceito deve abranger tanto os membros da equipe de saúde, quanto os pacientes que são os maiores interessados em receber assistência de qualidade ${ }^{(10)}$.

Nesse sentido, o cliente ocupar lugar de destaque é uma tendência da gestão contemporânea dos serviços de saúde, o que significa que a equipe necessita se preparar para lidar de maneira natural com esse usuário, que cada vez mais estará envolvido com o seu processo terapêutico e com o trabalho desenvolvido nas organizações, propiciando grande avanço no que se refere ao atendimento das reais necessidades da clientela atendida ${ }^{(11)}$

A maior média $(3,33)$ dos escores atribuídos foi encontrada na proposição 11 , com desvio padrão de 0,778 , onde as enfermeiras discordaram da seguinte afirmação: o treinamento dos colaboradores deve ser somente direcionado para as tarefas que irão desempenhar. Tal proposição enfatiza uma das características da Teoria Científica, preconizada por Taylor, que prioriza a tarefa e a especialização do trabalhador, visando aumento da produtividade.

Os princípios da Teoria Científica, historicamente, também foram incorporados pela enfermagem, visto que na prática assistencial há preocupação com o "como fazer", aliado ainda à divisão de trabalho que fragmenta a assistência ${ }^{(12)}$.

Temos observado, através da literatura, tentativa de ruptura com os princípios mecanicistas e reducionistas da Administração Científica, visto que atualmente novos valores estão influenciando a organização do trabalho e o mercado vem sofrendo transformações expressivas. Com o desaparecimento dos empregos, surgindo em seu lugar posições temporárias dentro das organizações, os trabalhadores não podem mais desenvolver somente tarefas restritas, mas, sim, devem ampliar seus conhecimentos, buscando a polivalência funcional, desempenhando novas tarefas $^{(13)}$. Parece-nos que as enfermeiras estudadas, ao discordarem da proposição 11 , estão em sintonia com essa nova forma de pensar, citada pelo autor.

Na proposição 18 que discorre sobre a valorização da hierarquia, enfatizando que mudanças no setor de trabalho devem partir sempre do escalão mais alto do serviço de enfermagem, encontramos a média de discordância de 3,176 e desvio padrão de 0,937. O princípio da hierarquia é um pressuposto da Escola Clássica idealizada por Fayol ${ }^{(4)}$, que preconiza as linhas de mando e autoridade, sendo que os administradores são as pessoas responsáveis pelo planejamento que repassam as ordens aos subordinados. Essa abordagem gerencial evidencia a estrutura formal da organização em detrimento da estrutura informal.

A partir do resultado obtido, constatamos que as enfermeiras discordaram da afirmativa proposta. Mais uma vez, podemos inferir que as enfermeiras compreendem que a estrutura formal muitas vezes pode reagir lentamente às mudanças, influenciando de forma negativa o andamento da organização, tornando morosa a resolutividade dos problemas cotidianos ${ }^{(12)}$. Nesse sentido, as participantes da pesquisa conseguem ultrapassar os limites da estrutura formal, buscando compreender a importância das relações informais.

Tendo em vista a proposição 20 em que a enfermeira deve desenvolver o seu trabalho obedecendo a esquemas rígidos e pré-estabelecidos, enfatizando a estrutura formal da organização, evidenciamos a média de 2,917 com desvio padrão de 0,900.

A discordância da proposição em questão nos leva a entender que as enfermeiras da instituição em estudo também estão buscando desenvolver sua criatividade no trabalho, haja vista que o desenvolvimento de tarefas padronizadas rigidamente através dos manuais de rotinas e procedimentos levam os indivíduos à mecanização do trabalho, gerando desmotivação e conseqüente insatisfação do trabalhador.

O cotidiano da enfermagem está cercado por uma 
série de dificuldades no desenvolvimento do seu trabalho, as quais se manifestam através da falta de motivação, insatisfação e falta de criatividade por parte dos trabalhadores, devido a alguns fatores, dos quais podemos destacar o desenvolvimento de tarefas limitadas e repetitivas ${ }^{(2)}$.

Atualmente os trabalhadores não querem ser apenas parte de uma engrenagem, mas sim pessoas ativas e participantes do processo de trabalho. Dessa forma, as pessoas inseridas nas instituições, inclusive aquelas da saúde, apresentam algumas necessidades como: interação pessoal, desenvolvimento da criatividade, valorização individual e do trabalho realizado, bem como humanização nas relações ${ }^{(14)}$.

\section{CONSIDERAÇÕES FINAIS}

Dada a importância de conhecer a conduta das enfermeiras em relação à sua equipe de trabalho, o presente estudo nos possibilitou identificar as atitudes que as enfermeiras de uma maternidade privada estão adotando diante de situações cotidianas relacionadas ao seu trabalho gerencial. Constatamos, através desta pesquisa, que há tendência à democratização das suas ações, uma vez que a maioria das respostas privilegiou questões acerca do trabalho em equipe, participação dos trabalhadores e desenvolvimento grupal, sem perder de vista os objetivos da organização. Reforçando essa atitude adotada pelas enfermeiras em questão, evidenciamos que grande parte delas discordou das proposições que abordavam os princípios mecanicistas e reducionistas, norteadores das Teorias Científica e Clássica da Administração, que influenciaram e ainda influenciam o

\section{REFERÊNCIAS BIBLIOGRÁFICAS}

1. Trevizan MA. Enfermagem hospitalar: administração \& burocracia. Brasília (DF): Universidade de Brasília; 1988.

2. Fávero N. O gerenciamento do enfermeiro na assistência ao paciente hospitalizado. [tese]. Ribeirão Preto (SP): Escola de Enfermagem de Ribeirão Preto/USP; 1996.

3. Trevizan MA. Liderança do enfermeiro: o ideal e o real no contexto hospitalar. São Paulo (SP): Sarvier; 1993.

4. Chiavenato I. Administração, teoria, processo e prática. $2^{a}$ ed. São Paulo (SP): Makron Books; 1994.

5. Médici AC, Silva PLB.Administração flexível: introdução às novas filosofias de gestão. RAP 1993 julho/setembro; 27(3):26-35.

6. Motta PR. Gestão contemporânea: a ciência e a arte de ser dirigente. $3^{a}$ ed. Rio de Janeiro (RJ): Record; 1993. trabalho da enfermagem.

Apesar de prevalecer na enfermagem uma raiz histórica de autoritarismo, centralização das decisões e impessoalidade nas relações, ainda presentes nos dias atuais, observamos que a introdução de novas abordagens gerenciais, devido às intensas transformações que estão ocorrendo na sociedade, está impulsionando os gerentes a buscarem novas alternativas para organizar o trabalho. Essas novas abordagens trazem no seu bojo conceitos de flexibilidade, redução da hierarquia, trabalho em equipe e descentralização das decisões, visando a satisfação dos clientes e trabalhadores, bem como a produtividade e a responsabilidade compartilhada, que devem ser vislumbradas pelos gerentes do futuro.

Consideramos que a introdução do gerenciamento da qualidade, na maternidade em estudo, sendo certificada com a ISO 9002, em março de 1999, pode ser uma variável que esteja influenciando sobremaneira a conduta das enfermeiras pesquisadas. Contudo, acreditamos ser esse um momento de transição em que as enfermeiras possam estar rompendo com alguns pressupostos tayloristas e fayolistas, tendo em vista perspectivas de mudanças na sua prática profissional. Entretanto, tal fato não pode ser afirmado com tanta convicção, uma vez que não tivemos a percepção do pessoal de nível médio acerca das atitudes adotadas pelas enfermeiras da instituição.

Novos estudos, portanto, deverão ser realizados a fim de nos permitir comparar as visões do gerente e seus colaboradores acerca das condutas adotadas na prática hospitalar, bem como trazer contribuições à profissão, entendendo que, para coordenar a equipe de enfermagem, a enfermeira deve assumir postura mais humanizada, acompanhando a velocidade das mudanças atuais.

7. Spagnol CA, Ferraz CA.Tendências e perspectivas da administração em enfermagem: um estudo na Santa Casa de Belo Horizonte-MG. Rev Latino-am Enfermagem 2002 janeiro/fevereiro; 10(1):15-20.

8. Fleury MTL. Desafios e impasses na formação do gestor inovador. In: Davel E, Vasconcelos J, organizadores. Recursos humanos e subjetividade. Petrópolis (RJ): Vozes; 1995. p.18796.

9. Bocchi SCM, Fávero N.Caracterização das atividades diárias do enfermeiro chefe de seção em um Hospital Universitário. Rev Latino-am Enfermagem 1996 julho; 4(2):4159.

10. Barros SMPF. Gerenciamento em saúde: implicações, tendências e perspectivas para a enfermagem. In: $45^{\circ}$ Congresso Brasileiro de Enfermagem; 1993. Recife, Pernambuco. Recife: Associação Brasileira de Enfermagem; 1994. p.93-103. 
11. Alves M, Carvalho H, Amaral MA.Fundação de Assistência Integral à Saúde/ Hospital Sofia Feldman: uma experiência de administração flexível?. Rev Latino-am Enfermagem 1999 outubro; 7(4):5-11.

12. Kurcgant $P$, coordenadora. Administração em enfermagem. São Paulo (SP): EPU; 1991.

13. Motta PR. Transformação organizacional: a teoria e a arte de inovar. Rio de Janeiro (RJ): Qualitymark; 1998.

14. Spagnol CA, Valle MSFR, Flório MCS, Barreto RASS, Sant'Ana RPM, Carvalho VT. O método funcional na prática da enfermagem abordado através da dinâmica de grupo: relato de experiência. In: $10^{\circ}$ Seminário Nacional de Pesquisa em Enfermagem; 1999. maio; Gramado; Rio Grande do Sul. Gramado: Associação Brasileira de Enfermagem; 1999. p. 203. 